\title{
Los maestros de Educación básica en México (D.F.) ante la reestructuración del sector educativo
}

\author{
José David Alarid Dieguez*
}

\section{Resumen:}

En el presente trabajo se abordan las formas en las que la reestructuración en el sector educativo en México que, se ha traducido en la implementación de políticas educativas "modernizadoras", ha incidido en la reconfiguración de las condiciones laborales de los maestros de educación básica en México y con ello se ha afectado la manera en que acometen su labor. La reestructuración en curso ha seguido las pautas marcadas por organismos internacionales como el BM y el FMI, destacadamente, entre otras, nuevas políticas de retribución diferencial del trabajo docente. Asimismo, se analizan las estrategias con las que los profesores de educación básica de la Ciudad de México han afrontado las consecuencias que ellos viven como negativas de la reforma educativa para su trabajo y la educación pública.

Palabras-clave: Educación-México. Reforma Educacional-México.

\footnotetext{
* Profesor da Universidad Pedagógica Nacional, Unidad Ajusco, México (D. F.). Licenciado en Sociología y en Psicología por la Universidad Nacional Autónoma de México. Maestro en Ciencias con especialidad en Investigaciones Educativas por el DIE - CINVESTAV - IPN. Candidato a Doctor en Sociología por la Universidad Nacional Autónoma de México (UNAM).
} 


\section{Introducción}

Este trabajo forma parte de una investigación en curso en la se pretende analizar las formas en las que a partir la "modernización" de la educación básica, con sus exigencias de eficiencia, productividad y calidad; se han visto reestructuradas las condiciones de trabajo de los maestros de México y cómo estas transformaciones estructurales han repercutido en la constitución de las formas en que los maestros significan su trabajo, su identidad y las respuestas a las transformaciones a través de la constitución de nuevas formas de representación en el trabajo, así como el cambio en las relaciones entre sindicato y Estado y su repercusión en las dinámicas y estructuras sindicales.

\section{El problema}

En la época de la llamada sociedad del conocimiento la producción, divulgación y enseñanza de conocimiento se consideran fundamentales para el desarrollo social de cualquier país. En ese contexto la labor de los profesores y académicos adquiere especial relevancia para desarrollar las tareas que tiene que ver con la producción, divulgación y enseñanza de conocimiento, por lo cual su trabajo se mira cada vez más con especial atención pues se considera que a partir de él se pueden impulsar los procesos modernizadores en la sociedad mexicana.

México, como toda América Latina, se ha visto inmerso en un proceso de reestructuración social desde una perspectiva globalizadora de corte neoliberal que, bajo la inspiración de organismos transnacionales, ha impuesto pautas de reorganización social que han conformado la reestructuración del modelo de acumulación. La crisis fiscal de los estados latinoamericanos, agudizada por la carga de la deuda externa alentó a que los gobiernos de América Latina asumieran las directrices de los organismos internacionales como el Banco Mundial (BM) y el Fondo Monetario Internacional (FMI) pensadas para desregular a las sociedades y abrirlas las economías de la región a las formulas del libre comercio (BONAL, 2002), México no ha sido la excepción.

Que las reformas educativas en América Latina han sido inspiradas por el FMI y el BM es de sobra conocido (BONAL, 2002) sin embargo 
eso no significa que todos los países de la región hayan seguido una senda única. En cada país se han implementado las reformas de acuerdos a las condiciones políticas y sociales prevalecientes según la configuración del bloque histórico prevaleciente.

Las reformas se han implementado en contextos de austeridad presupuestal y con propósitos que incluyen el recorte del gasto presupuestal, concretado, entre otras cosas, en bajas en los salarios reales. Así, las remuneraciones magisteriales han disminuido de manera significativa desde la década de los 80 .

Las reformas educativas se han planteado como metas: mayor equidad de acceso, mejora de la calidad y resultados, sistemas de rendición de cuentas, revisión del currículo y de contenidos y de textos.

En 1990 el Banco Mundial publicó un estudio sobre educación básica titulado; "la educación primaria. Documento de política", el que se constituyó en un referente de algunas políticas entre las que destacan la necesidad de revisar los libros de texto, la propuesta de evitar incrementos salariales que no estuvieran vinculados a un análisis de resultados, la justificación para establecer un proyecto escolar, la propuesta de descentralizar el sistema educativo (DÍAZ BARRIGA, 1999). Recomendaciones adoptadas una a una por el Estado mexicano y que tuvieron en su mayoría concreción en el Acuerdo Nacional para la Modernización de la Educación Básica (ANMEB).

Las reformas se han dado con vistas abordar problemas de financiamiento, competitividad y equidad. Ante las crisis económicas ha habido disminución del gasto educativo, aunque contradictoriamente una presión para aumentar las matrículas, también se ha intentado responder a la mundialización, incrementando el 'rendimiento escolar' y la habilidades laborales con vistas a preparar capital humano de 'más calidad' que permita mejorar la competitividad a nivel mundial y también se intentó favorecer el papel de la educación como fuente de movilidad social. Se pretende descentralizar para mejorar la escuela y economizar recursos. En las reformas se concede un gran valor a los indicadores formales, incremento de la matrícula, de índice de aprobación, etc.

En el sector de la educación esto se ha traducido en una reorganización del sector y se pusieron en marcha una serie de recortes a los presupuestos destinados a la educación para responder simultáneamente a políticas de 
austeridad y a una reorganización del sector educativo para que responda a las nuevas exigencias del mercado de trabajo.

Sin duda, el sector educativo tiene una importancia primordial en el establecimiento y mantenimiento de la hegemonía, a través de participar en la aculturación de importantes sectores de la población en pautas de socialización que son marcadas en el currículo oficial de la educación básica en México.

Para esto se ha puesto en marcha a partir de la década de los 80 y en especial desde 1992 de un proceso de reestructuración del sector a través de una serie de medidas entre las que destacan: la federalización de los servicios educativos a los estados de la federación (con la excepción del D. F.), el reforzamiento de la capacidad normativa del gobierno federal vía la Secretaría de Educación Pública (SEP), que conserva la facultad de decidir sobre el currículo de la educación básica en el nivel nacional, la implementación de una serie de programas de impacto en la transformación del sector y, la puesta en marcha del programa denominado Carrera Magisterial como eje que vertebra la reconfiguración del trabajo magisterial.

Si bien existen una serie de estudios que muestran algunos efectos de la puesta en marcha de estas medidas para el magisterio (JIMÉNEZ, 2003; ALARID, 2001) hace falta explicar y comprender el proceso de reestructuración de la labor docente desde la reestructuración organizativa de aquel, de tal manera que se puedan ubicar los efectos desde una perspectiva más amplia (SMYTH, 2000).

Asimismo, consideramos pertinente realizar estudios regionales que den cuenta de las particularidades de la reestructuración en distintas zonas del país, atendiendo a las importantes diferencias en las condiciones en que se desarrolla el trabajo magisterial, sin menoscabo de atender sus bases comunes.

Interesa también, y de manera preponderante, estudiar el proceso desde la perspectiva de los propios maestros como sujetos sociales que con su acción cotidiana constituyen al hecho educativo en su concreción. Esto implica abordar la problemática de la reestructuración educativa situando a los maestros no como simple ejecutores de planes prescritos desde las instancias de gobierno, sino como actores que dependiendo de su posición ante el proceso juegan en la conformación de los resultados educativos. Tradiciones, cultura, posiciones políticas y pedagógicas, etc., son elementos 
desde los cuales los maestros conforman su acción. De tal manera que ante las propuestas oficiales los maestros pueden responder con aceptación, rechazo, resistencia, contrapropuestas, etc.

Las respuestas de los maestros se dan en distintas direcciones y en formas desde las más individuales hasta aquellas que se organizan en pautas colectivas de acción, desde las de nivel escuela hasta las que se han expresado en el nivel de su sección sindical.

Se analizan las consecuencias del proyecto modernizador en las condiciones laborales y de representación de los maestros de del D. F. Los maestros de educación básica agrupados en la sección 9 del Sindicato Nacional de Trabajadores de la Educación (SNTE) constituyen el sujeto de análisis del presente trabajo de investigación.

\section{La reforma de la Educación básica}

Los gobiernos posrevolucionarios mexicanos, tanto los de origen priista como el actual de raigambre panista, han basado una parte importante de su discurso legitimador en la promoción del aumento de indicadores de bienestar de la población referidos al acceso de sectores cada vez mayores de ella a servicios como la salud, la vivienda, el trabajo y la educación.

Sin embargo, en el sector educativo subsisten rezagos y carencias importantes, a pesar de que, cuando menos desde la época del presidente A. López Mateos (1958-1964) se han emprendido una serie de reformas educativas destinadas a 'mejorar' la educación. En el sexenio actual se han mantenido las líneas 'modernizadoras' trazadas en el sexenio del presidente Carlos Salinas (1988-1994) y continuado en los de Ernesto Zedillo (19941996) y Vicente Fox (2000-2006).

La reforma educativa en México debe ser ubicada en la perspectiva de las transformaciones sociales que a partir de la década de los 80 se pusieron en marcha en la mayor parte de América Latina. Los gobiernos de la región cambiaron su estrategia de desarrollo desde la visión de la sustitución importaciones hacia el modelo 'neoliberal'. La puesta en marcha de este proceso se dio en un contexto de baja una fuerte crisis económica derivada del peso de la deuda externa para las finanzas estatales (BONAL, 2002). El sector educativo se ha visto sujeto a las directrices políticas derivadas de la puesta en marcha del modelo de desarrollo de libre mercado, orientado a la exportación. 
Así una serie de orientaciones de la política educativa se han dirigido a entre otras cosas a 'eficientar' el gasto educativo, 'hacer más con menos' ha sido uno de los lemas favoritos que han guiado las reformas. La reforma educativa modernizadora fue diseña para adecuar el sector educativo a la política de reestructuración social planteada desde el gobierno de Carlos Salinas. ${ }^{1}$

Cabe hacer notar que el discurso empresarial ha ido ganando lugar en la educación básica en México y ha influyendo los discursos educativos; veremos algún caso de la utilización de estos en el contexto nacional. ${ }^{2}$

A continuación plantearemos las líneas generales de la modernización educativa planteada en el Acuerdo Nacional para la Modernización de la Educación Básica, las cuales siguen rigiendo el proceso de reestructuración del sector educativo en el país.

\section{El Acuerdo Nacional para la Modernización de la Educación básica}

El sexenio de Carlos Salinas de Gortari (1988-1994) representó para el país la puesta en marcha de una serie de políticas de transformación estructural en los distintos órdenes: económico, político y social. En cada sector se emprendieron cambios dirigidos a lograr la transformación de México hacia ser una nación 'moderna', o más correctamente dicho, hacia una cierta visión de la modernización a la que se ha calificado de manera reiterada como 'neoliberal'. (DE LA GARZA, 2000).

Entre los objetivos que adoptan los gobiernos que siguen esa orientación destacan el 'adelgazamiento del Estado'. De esa forma, en un contexto de austeridad presupuestal los servicios sociales prestados por el gobierno mexicano se han visto sujetos a las políticas 'eficientizadoras', que se traducen en la tendencia al recorte de los gastos destinados a los rubros sociales.

En este contexto, podemos ubicar que en el sector educativo se impulsaron una serie de programas destinados a poner a tono a ese sector con el resto de los cambios.

En 1992 se firmó el Acuerdo Nacional para la Modernización de la Educación Básica (MÉXICO, 1992) en donde se concretó de manera más precisa el plan modernizador sexenal para el sector de la educación básica 
del país y que ha continuado como el referente para las políticas públicas para el sector. ${ }^{3}$

En ese documento se afirma que una serie de propósitos como 'una economía nacional en crecimiento y con estabilidad, y una organización social fincada en la democracia, la libertad y la justicia', son objetivos que 'exigen una educación de alta calidad, con carácter nacional y con capacidad institucional que asegure niveles educativos suficientes (sic) para toda la población. Asimismo, se afirma que la estrategia de 'modernización' del país y la reforma del Estado 'requieren que se aceleren los cambios en el orden educativo’ (MÉXICO PODER EJECUTIVO FEDERAL, 1992, p. 1).

Para El Gobierno se hacía necesario transformar el sistema de educación básica con el propósito de convertirla en la palanca de desarrollo individual y social.

El ANMEB fue firmado por el Gobierno Federal, los gobiernos estatales de la república y el Sindicato Nacional de Trabajadores de la Educación con el compromiso de: extender la cobertura de los servicios educativos, elevar la calidad de la educación, uso de recursos presupuestales crecientes para la educación pública, reorganización del sistema educativo, reformulación de los contenidos y materiales educativos y la revaloración de la función magisterial.

En el documento se afirma,

La calidad de la educación básica es deficiente en que, por diversos motivos, no proporciona el conjunto adecuado de conocimientos, habilidades, capacidades y destrezas, actitudes y valores necesarios para el desenvolvimiento de los educandos y para que estén en condiciones de contribuir, efectivamente, a su propio progreso social y al desarrollo del país.(MÉXICO PODER EJECUTIVO FEDERAL, 1992, p. 5).

es decir, si bien se reconocen las grandes limitaciones que imperan en la educación básica, se elude buscar responsables de tal situación'. ${ }^{4}$ Se afirma que la escasez de recursos: limitó el quehacer educativo y la capacidad de 'motivar' al magisterio. El Acuerdo significó que el Ejecutivo Federal traspasara a los gobiernos estatales, los establecimientos escolares con sus elementos de carácter técnico y administrativo, derechos y obligaciones, 
bienes muebles e inmuebles, de igual forma, asimismo traspasó los recursos para operar tales planteles educativos.

En el Acuerdo quedó que los gobiernos estatales reconocen al SNTE como el titular de las relaciones laborales colectivas de los trabajadores de base que se incorporan al sistema educativo estatal. ${ }^{5}$ También, se hace hincapié en favorecer 'la nueva participación social', para lo cual considera importante fortalecer la capacidad de organización y la participación desde las escuelas de maestros, padres de familia y alumnos. Ahí está en ciernes ya la implementación de un dispositivo control de padres de familia sobre la labor de los docentes, de forma tal que se garantice el cumplimiento de planes y programas educativos. ${ }^{6}$

Por último, el Acuerdo se ocupa de la Revaloración de la Función Magisterial, se afirma que el protagonista de la 'transformación educativa' debe ser el maestro. Él cual es caracterizado como quien transmite los conocimientos, fomenta la curiosidad intelectual y 'debe ser ejemplo de superación profesional' (MÉXICO PODER EJECUTIVO FEDERAL, 1992, p. 17). Se asume que dado que el maestro es quien mejor conoce las debilidades y virtudes del sistema educativo, sin su concurso, cualquier intento de reforma se vería frustrado. De ahí que, 'uno de los objetivos centrales de la transformación educativa sea 'revalorar la función del maestro'.

En el documento se anuncia la creación de la llamada Carrera Magisterial (CM) Tal vez, la parte más importante del Acuerdo, junto con la descentralización, en términos de sus consecuencias para el sector. Tal programa se anuncia con el fin de impulsar el 'arraigo y motivación' del maestro y, como una respuesta a la demanda del SNTE.

Se señala que la CM daría respuesta a dos necesidades de la actividad docente: "estimular la calidad de la educación y establecer un medio claro de mejoramiento profesional, material y de la condición social del maestro." (MÉXICO PODER EJECUTIVO FEDERAL 1992, p. 20). Se le plantea como un mecanismo de 'promoción horizontal' para el personal docente frente a grupo de los ciclos de educación básica. El propósito de la carrera magisterial sería que los maestros frente a grupo pudieran acceder a niveles salariales 'superiores' con base en "su preparación académica, la atención a los cursos de actualización, su desempeño profesional, y su antigüedad 
en el servicio y en los niveles de la propia carrera magisterial." (MÉXICO PODER EJECUTIVO FEDERAL, 1992, p. 21).

En términos de las transformaciones de trabajo de los maestros, la carrera magisterial representa un hito importante por las consecuencias de la implementación de este programa en las actividades cotidianas de los maestros de educación básica y en su relación con su sindicato. ${ }^{7}$

El documento concluye así:

\begin{abstract}
Asistimos a un momento decisivo de nuestra historia en el que el cambio es el signo del tiempo. Los mexicanos hemos decidido modernizar nuestro país. La transformación educativa se inscribe en esa estrategia. Con ella daremos respuesta a las necesidades del futuro con el ritmo que demanda el mundo contemporáneo, y aseguraremos una modernización que fortalezca nuestra identidad nacional en el marco de una creciente prosperidad general. (MÉXICO PODER EJECUTIVO FEDERAL, 1992, p. 21, bastardillas mías). ${ }^{8}$
\end{abstract}

Podemos ubicar el que la CM aparece como un plan de reestructuración de las condiciones de trabajo y promoción de los profesores, que transformaría las viejas formas de gestión de la fuerza de trabajo magisterial.

La descentralización fue una política central de las reformas implementadas y a partir del ANMEB se transfirieron los servicios educativos a los estados de la república, con la excepción del D. F., asiento de la capital política del país. Gobernada desde 1997 por el Partido de la Revolución Democrática (PRD), partido de centro izquierda y principal opositor del oficialista Partido Acción Nacional (PAN). Las causas de esa situación son abiertamente políticas; un contingente sindical con una posición opositora activa como lo fue la sección 9 del SNTE en la capital del país resulta un elemento indeseable para el bloque hegemónico. Como en el D. F. la transferencia de los servicios educativos al Gobierno del Distrito Federal. no se ha realizado, los efectos de la descentralización no se han hecho sentir. Con la consolidación en el Gobierno del D. F. de una administración de oposición al partido del jefe del ejecutivo, que lleva ya casi 11 años en el poder se plantea la posibilidad de un proceso en el que 
los maestros de la Sección 9 puedan negociar algunas de las condiciones del proceso de descentralización, de forma tal que no les sean desfavorables.

\section{La carrera Magisterial y la evaluación en la Educación}

La evaluación se ha convertido en una de las piedras de toque del funcionamiento del sistema de regulación del trabajo docente (DÍAZ BARRIGA, 1999). La descentralización educativa no significó la descentralización en la elaboración de planes y programas educativos; por el contrario, se explícito que es facultad del ejecutivo federal, a través de la SEP de elaborar los planes y programas de estudio vigentes en la educación básica. Esto supone que en lo general, el trabajo cotidiano de los docentes de educación básica está regulado por directrices emitidas centralmente desde la SEP que se concretan en el currículo oficial de la educación básica y otros ordenamientos. En esa perspectiva es que situamos al establecimiento de la evaluación como uno de los elementos centrales de la implementación de los procesos de reestructuración del trabajo docente derivados de la modernización educativa.

El establecimiento de la CM ha significado un impulso en esta dirección en la medida en que una de las vertientes de evaluación de los profesores que se establece en los lineamientos de CM para lograr ingreso y/o promociones, es el resultado promedio de exámenes aplicados muestras de alumnos de los profesores para evaluar su aprovechamiento. Con ese indicador se intenta medir la 'eficiencia' en el trabajo docente. Un propósito de esa condición es intentar estandarizar la labor de los maestros no sólo en tiempos sino también en contenidos, dado que para poder obtener los puntos asignados en ese rubro el maestro necesita programar sus actividades de tal forma que se sincronicen con las evaluaciones programadas, lo que implica que su programación se sujeta a tiempos externos más que atender al ritmo de aprendizaje de sus alumnos, lo cual desde el punto de vista psicopedagógico, es lo óptimo para poder favorecer el desarrollo de aprendizaje de tipo 'significativo'. (COLL, 1990). Esta situación le supone al docente aumento de estrés y sobrecarga de trabajo. Sus criterios se vuelven más cuantitativos que cualitativos en la evaluación de su trabajo y del desempeño de sus alumnos.

Por otro lado, los resultados de las evaluaciones se utilizan como indicadores de desempeño para diferenciar a los 'buenos' de los 'malos' 
maestros, sin que necesariamente esos resultados reflejen, desde la perspectiva de los profesores, la calidad de la labor desarrollada (ALARID, 2001). Otra fase de la evaluación de los maestros es la que se desarrolla entre los miembros del centro de trabajo (MÉXICO SECRETARIA DE EDUCACIÓN PÚBLICA, 1998), en la que el director de la escuela tiene un peso mayor que el de los maestros (ALARID, 2001). . La competitividad generada entre los maestros tiende a generar más angustia y aislamiento. ${ }^{10}$

La carrera magisterial tiene como resultado la diferenciación de las remuneraciones que perciben los maestros. A la fecha existen cinco niveles de ingresos: sin ingresar a CM, niveles a, b, c y d. Las diferencias de ingresos salariales entre cada uno de estos niveles es de 33\%, aproximadamente; por lo cual un profesor que se encuentre en el nivel "c" percibirá el doble que lo recibe un profesor que no ha ingresado al programa. Sin duda que esto marca notables diferencias de ingresos entre quienes componen la planta docente de las escuelas.

Es de hacer notar que se comenta entre los profesores que en distintos momentos de los procesos de evaluación existe corrupción. Por ejemplo, se dice que los exámenes se venden en el "mercado negro" y que hay quienes los consiguen y obtienen ventajas sobre quienes los presentan sin recurrir a esa artimaña. Esta situación supone para ellos un elemento de descalificación de la objetividad pretendida en la evaluación y, por tanto, su descalificación. ${ }^{11}$ Lo que aunado a inconformidades respecto de las formas en que los órganos de evaluación, presididos por los directores de las escuelas suelen evaluar y, por tanto asignar puntos, que incide en la calificación total y en las posibilidades de ingresar y/o promoverse en la CM podemos afirmar, con base en las entrevistas realizadas, que para un sector importante de los docentes la CM no refleja la calidad de la labor efectuada en el día a día y lo interesante es que esto es opinado aún por profesores que están en niveles altos, "C" o "D", de CM y que, por tanto, pueden ser considerados como los 'ganadores' de la situación.

Sin embargo, en otros casos quienes sí están en los niveles altos suelen considerar la situación como indicador de sus mayores méritos; lo que en algunos casos lleva a tensiones entre los miembros del personal docente y han determinado divisiones, lo que dificulta el trabajo al interior de las escuelas. 
Cabe hacer notar que el programa de CM tiene límites presupuestales por lo cual el acceso a la misma está dado por los techos presupuestales asignados. Esto significa que el criterio de acceso es de cupo y no de puntajes, es decir ingresan tantos docentes como la bolsa asignada lo permita.

Analistas del proceso como S. Street reportan que la CM impone nuevas condiciones de trabajo al fomentar la intensificación, apunta simultáneamente a aumentar el control central de la materia de trabajo del docente y a minar la identidad gremial y profesional de los maestros. De igual manera, los saberes docentes son puestos en cuestión y se intenta implementar una nueva racionalidad al trabajo del profesor (1998). En ese clima se vuelve más difícil llegar a acuerdos colectivos y después a sostenerlos.

Con lo hasta aquí planteado al respecto de CM es posible adelantar que las consecuencias de ese programa rebasan, por mucho, las afirmaciones de que, tal programa, es una forma de lograr 'mejorar' la calidad de la labor docente, al mismo tiempo que de mejorar las remuneraciones económicas de los maestros.

En esta perspectiva cabe señalar que en mayo de 2008 la SEP anuncia estímulo por desempeño, con lo que se pretende recompensar a los profesores cuyos alumnos logren mejoras en sus evaluaciones nacionales. Esta medida revela de manera nítida la concepción que del desempeño docente sostienen el Estado y las dirigencia sindical, quienes acordaron tal medida; labor destinada a producir un producto predeterminado sin que se valoren las diferencias de contextos en los que se produce el hecho educativo. Promoviéndose, además, efectos perversos como, la puesta en marcha de estrategias encaminadas a maximizar las posibilidades de obtener ingresos adicionales por parte de algunos maestros, sin que esto signifique "mejora de la calidad" educativa.

\section{Los Maestros y "su" Sindicato, el SNTE}

El SNTE es el sindicato que agrupa a los trabajadores de la educación que prestación sus servicios en dependencias educativas oficiales no autónomas. Es el sindicato más grande de América Latina por su número de afiliados de más de 1.200,000 agremiados. El poder de su dirigencia ha sido muy importante dentro del sistema político mexicano (ARNAUT, 
1996)..$^{12}$ El SNTE monopoliza la representación de los maestros del país y la Secretaría de Educación Pública lo reconoce oficialmente como único interlocutor legal para tratar los asuntos del magisterio.

En lo que respecta a los maestros de educación básica que analizaremos pertenecen a la sección 9 del SNTE y han sido desde 1989 parte de la disidencia sindical a la corriente hegemónica en el sindicato. En 1979 surge la Coordinadora Nacional de los Trabajadores de la Educación (CNTE), como expresión de descontento con el liderazgo corporativo que había prevalecido en el SNTE desde su creación. La corriente hegemónica en el sindicato en ese momento, la autodenominada Vanguardia Revolucionaria del Magisterio la combate de manera frontal utilizando sus múltiples recursos, desde los que les permite el uso discrecional de unos estatutos diseñados ad hoc para el control del sindicato por parte de una minoría, hasta formas de hostigamiento extralegales (COOK, 1996). ${ }^{13}$

En 1989 se desarrolló un movimiento magisterial masivo, en semanas un contingente que no participaba masivamente desde las luchas magisteriales de 1958, la Sección IX del SNTE que agrupa a los maestros de educación preescolar, primaria y especial en el D.F. se incorporó a la lucha por $100 \%$ de incremento salarial y democracia sindical. Se calcula que en el momento de máximo auge del movimiento en mayo de ese año, estuvieron movilizados, bajo distintas modalidades, casi 500,000 trabajadores de la educación.

Aprovechando la coyuntura abierta por el movimiento desde las cúpulas del sistema político mexicano se decide la salida del grupo de hegemónico en el sindicato durante 17 años y en su lugar se nombra a Elba Esther Gordillo (EEG) como nueva secretaria general del SNTE. Se termina la hegemonía de un grupo que llegó a ser muy poderoso dentro del sistema político corporativo mexicano (STREET, 1992) y se instaura un liderazgo que pretendió ser 'más moderno'. ${ }^{14}$ La visión del grupo consolidado periodo presidencial 1970-1976 representaba un obstáculo a la modernización del sector educativo, desde el punto de vista de la nueva élite en el poder. ${ }^{15}$

En la coyuntura actual el CEN SNTE mantiene el control del sindicato, pero ha perdido su poder negociador en otros rubros (STREET, 2001). 
Con la situación de deterioro salarial ha que se han visto sometidos los trabajadores de la educación, existe una erosión de las bases de legitimidad que el SNTE mantiene ante ciertos sectores de la 'base magisterial'.

Por otro lado es necesario decir que, si bien entre el Ejecutivo Federal y la dirección del SNTE subsisten diferencias de criterios al respecto del proceso de modernización; tales diferencias son negociadas al interior del sistema (neo) corporativo, por lo cual, hacia el exterior la dirigencia del SNTE aparece negociando prebendas a cambio del apoyo, en lo fundamental, las políticas gubernamentales.

Por su lado, los maestros disidentes agrupados en la CNTE se manifiestan como contrarios a las políticas modernizadoras de inspiración 'neoliberal' que atraviesan al sector.

Sin haber logrado una alternativa viable a ellas y sin profundizar en el análisis del proceso más allá de sus manifestaciones más visibles, se han planteado la necesidad de 'resistir' y contrapesar las medidas que se consideran contrarias a los 'intereses del magisterio'.

Para poder organizarse más allá de la camisa de fuerza que representan los estatutos, los maestros 'disidentes' han implementado formas organizativas propias (COOK, 1996). Estas nuevas formas de representación sindical (NFRS) han permitido a los maestros, en momentos de movilización, implementar sus decisiones de manera directa, sin tener que pasar por la anuencia de la corriente hegemónica.

Podemos caracterizar al ideal de democracia imperante en la sección 9, como en los otros contingentes democráticos del SNTE, secciones 18 y 22 , en los momentos de vida sindical activa, con participación de los maestros como una democracia de tipo directa. ${ }^{16}$

Las NFRS han sido activadas por los maestros, en la medida en que las formas de representación sindical codificadas en los estatutos del SNTE responden a una idea centralizadora del poder sindical hacia la 'elite' dominante y, por tanto, que 'ahogan' el poder de decisión de la base. Tal idea centralizadora responde a las formas de control que se han desarrollado en el SNTE de manera histórica.

Quisiéramos resaltar que es necesario pasar a un nivel de análisis más cercano a las opiniones o 'conciencia discursiva' de los profesores, en el lugar de trabajo. De tal manera que podamos tener fuentes de primera 
mano para comprender las formas de significar el proceso modernizador por parte de los profesores.

Afrontar de manera organizada las situaciones adversas a los intereses del magisterio requiere de la acción colectiva, la cual ha sido obstaculizada de manera sistemática por la cúpula del sindicato. Y no sólo eso; de hecho se ha mostrado opuesta a los intereses de sus representados.

Así, la dirigencia del SNTE ha sido una de las principales promotoras de las reformas a la ley del Instituto de Seguridad Social y Servicios de los Trabajadores del Estado (ISSSTE), a contracorriente de los deseos de la gran mayoría de los agremiados del sindicato que lidera, EEG se convirtió una de las mas importantes impulsoras de la iniciativa, con base en una alianza con otros "dirigentes" que había negociado posiciones ventajosas de poder en la administración de la de fondos de retiro que manejará las aportaciones de los trabajadores al servicio del Estado.

La reacción de los trabajadores de base del SNTE y de otros sindicatos ha sido de resistencia ante una medida que sin duda vulnera derechos y que se traduce en mayores cargas financieras para los trabajadores y menores expectativas de remuneración al momento de la jubilación, así como perdida de prestaciones, en suma, perdidas a cambio de ganancias para las camarillas dirigentes, quienes sacrifican a sus agremiados y sus intereses desde el discurso de el sacrificio es en nombre de los intereses superiores del país.

No solo eso, EEG proclama que se convertiría en una activa promotora de las "reformas estructuras", léase la energética y la laboral.

Ante estas posiciones de su "líder" importantes sectores del magisterio han respondido rechazando la reforma a la Ley del ISSSTE, que llevo a importantes movilizaciones en contra de su promulgación y, una vez promulgada, se ha formado un proceso de resistencia por la vía de los amparos. Que suman miles, hasta febrero de 2008 no se conocían con exactitud la cantidad de ellos, paro algunos los calculaban en el orden de los cientos de miles.

Ante lo cual, EEG reúne a sus huestes y les recrimina que no hubieran podido controlar a las bases del sindicato que han participado de manera activa en la presentación de amparos. En particular, le irritaba la masiva participación de los maestros de los estados del norte de la republica, tradicionalmente no activos en contra de las decisiones del SNTE. 
En el caso de los maestros del D. F. su participación en las movilizaciones se reactivo, en particular en la magna marcha y paro que realizaron en la víspera de la discusión del la ley que llevo a su aprobación registro una inusual participación de la base, que había permanecido inmóvil por mucho tiempo.

Posteriormente, han participado activamente en la presentación amparos, en contra de la ley citada.

El descontento generado por las reformas aun en sectores que no habían mostrado descontento contra la dirigencia del SNTE, ante su papel no sólo como promotor sino como abierto ganador de privilegios económicos en contra de los intereses de sus afiliados a los que, por supuesto, no consultó. Esta situación ha generado gran deslegitimación de la dirigencia del SNTE, la que cuenta con el aval estatal para mantenerse en el poder.

Ante esta situación, la cuestión de la legitimidad de la dirección del SNTE se avizora como cada vez más frágil, pero la dirección del sindicato sigue siendo sostenida por el Estado mexicano. De manera clara en algunas entidades del país, siendo los casos más visibles las secciones sindicales de Michoacán y Oaxaca existe un abierto rechazo mayoritario a la dirigencia del sindicato, en el D. F. sección 9 existe un gran rechazo sin que se manifieste como movilización.

Ante las reformas y sus efectos los maestros de educación básica del D. F. agrupados en la sección 9 del SNTE se han visto obstaculizados para enfrentar de manera organizada la situación, vivida por la mayoría de ellos como de deterioro de sus condiciones laborales y sus prestaciones.

La participación de los maestros ha sido bloqueada sistemáticamente por el Comité Ejecutivo Nacional (CEN) del SNTE; ante lo cual la mayoría de las respuestas que han podido articular ha sido principalmente individual y defensiva. Podemos afirmar que la representación legal que ostenta la cúpula representa una expropiación de la posibilidad de auto representarse y como indicador de esto cabe apuntar que en la sección 9 del SNTE no ha habido asambleas para renovar sus órganos de dirección por más de 10 años, lo que contraviene los estatutos del propio sindicato y, ante lo cual legalmente no han logrado ganar ese derecho. 


\section{Resultados de las Políticas Educativas en México}

En este parte haremos un breve balance de los resultados obtenidos de la puesta en marcha de las los políticas educativas.

Inspirados en los exámenes del Programa para la Evaluación Internacional de los Estudiantes (PISA) auspiciados por la Organización para la Cooperación y el Desarrollo Económicos (OCDE) se creó la prueba Evaluación Nacional del Logro Académico en Centros Escolares (ENLACE) que mide el rendimiento de los alumnos de educación básica. $\mathrm{Al}$ respecto cabe mencionar que, las reformas emprendidas medidas por sus resultados en evaluaciones internacionales como PISA de la OCDE están muy lejos de poder ser catalogadas como exitosas. Consistentemente las evaluaciones colocan al rendimiento de los estudiantes mexicanos entre los últimos lugares de la OCDE, lo que después de más de 15 años de que se firmó el Acuerdo Nacional Para La Modernización De La Educación Básica y Normal supone que no se han cumplido los objetos que se pretendían alcanzar.

Esto sin que esto implica que aceptemos la concepción que sobre educación impulsa el Estado mexicano.

La evaluación de los distintos aspectos de la educación ha sido uno de los más importantes referentes de las reformas. Evaluación encaminada a aumentar la eficacia y la eficiencia del gasto público y en la perspectiva de recortar al máximo posible los gastos sociales y desde una perspectiva que apuesta por la privatización de amplios espacios de la educación pública.

Cabe hacer resaltar que el diseño de las evaluaciones se ha dado sólo con la participación de la dirigencia del SNTE y sin la participación de los profesores de base.

Si bien no hay duda que la evaluación es un recurso necesario para poder tener control sobre los procesos educativos, el dilema se plantea en qué evaluar, de qué manera y quién lo hace. En el enfoque dominante en América Latina y que es el que prevalece en México se evalúa con base en el rendimiento de los alumnos, partiendo del supuesto que la acción docente sería el factor principal de tal rendimiento.

La perspectiva que se ha impuesto desde los centros de irradiación del pensamiento neoliberal sitúa a la evaluación como instrumento de 
obtención de datos que permitan el control del personal docente para poder administrar su trabajo de una manera más "eficiente" en la perspectiva de de estratificarlos y diferenciar el pago que se les hace, legitimando, al mismo tiempo, la exclusión de muchos de ellos de ingresos adecuados. En mayo de de este año se anuncia que se establece en México un estímulo para aquellos maestros cuyos grupos muestren una mejoría en sus resultados en la prueba ENLACE en relación con los resultados obtenidos por los alumnos en el ciclo escolar próximo pasado. Sin duda esta situación abre posibilidades a un cúmulo de efectos no deseados, pero previsibles como el que los docentes busquen obtener el estímulo.

\section{Notas}

1 También para intentar contrarrestar las condiciones generadas por la pérdida de legitimidad del SNTE, que se expresó en movimiento magisterial de 1989, en términos de la no-subordinación de importantes sectores magisteriales a las políticas gubernamentales.

2 La aportación de Schmelkes (1997) constituye un buen ejemplo de la manera en que el discurso de la "calidad total" se utiliza para intentar modificar las prácticas educativas en las escuelas públicas de México. El libro referido se ha constituido en una referencia básica de los modernizadores en la discusión acerca de la calidad educativa y las maneras de implementarla. Es posible ubicar de manera clara los tópicos clásicos del discurso empresarial en la obra citadaLo resaltamos es por su representatividad, de hecho fue impreso de manera masiva y difundida en la biblioteca del maestro, lo que significa que se le considera desde la SEP como una obra de referencia.

3 Se necesita realizar el análisis de los programas educativos de los gobiernos de Ernesto Zedillo y el actual, par situar los cambios de rumbo y las maneras específicas de implementación del proceso modernizador.

4 Lo que supone reafirmar una vieja práctica del discurso oficial; no responsabilizar directamente a los gobiernos anteriores de las situaciones actuales.

5 Hasta el momento la única entidad en la que no se ha concretado el ANMEB en el D. F. por lo cual el análisis de esta entidad puede servir 
como elemento de contraste con lo que ha pasado en los distintos estados del país. Los trabajos de Jiménez (2003) y el Street (2000) analizan proceso en la región de La Laguna en el estado de Coahuila y en el estado de Michoacán respectivamente y muestran algunos elementos comunes con la problemática del D. F. que nos proponemos analizar. Al respecto se puede decir que ha habido negociaciones entre las autoridades del Gobierno del Distrito Federal y Gobierno Federal para negociar las condiciones del traspaso de los servicios educativos, sin que hasta la fecha se haya concretado tal traspaso. Aunque ya por motivos políticos, restarle presupuesto al gobierno del D. F. se ha iniciado el proceso de descentralización.

6 Otro de los puntos importantes del Acuerdo es el relativo a la reformulación de los contenidos y materiales educativos.

7 Se intenta con este programa poner a los maestros ante perspectivas de promoción más individualizadas, con lo cual la movilización colectiva podría verse como menos atractiva Contamos ya con algunos elementos empíricos que nos permiten ubicar cómo ha sido utilizada por los maestros y cómo la han incorporado a sus reivindicaciones, aunque se hace necesario avanzar en un conocimiento más preciso y profundo de ese proceso. (ALARID, 2001).

8 El último párrafo muestra claramente como la globalización es el trasfondo de los procesos de cambio en educación. Es de notar la idea de que lo que venía en el país era una 'creciente prosperidad general'. Esta idea se desvaneció con furia en diciembre de 1994 cuando se desató la peor crisis económica en el siglo pasado. La promesa de bienestar resultó ser una ilusión que tuvo grandes costos para la mayoría de la población de México. Lo que resta es analizar las consecuencias del plan modernizador.

9 Esta situación ha significado que la evaluación pueda estar mediada por las relaciones interpersonales entre los maestros, lo que llega a generar fricciones entre los maestros y en ciertos casos divisiones entre los mismos; y por otro lado, tiene que ver con las relaciones de poder con el director de la escuela, lo que suele apuntalar las prácticas clientalares entre los maestros de banquillo y las autoridades escolares. 
10 Esta parece ser una tendencia general del trabajo docente en diversos países como E.E.U.U. y Canada. (HARGREAVES, 1996).

11 Situación que fue corroborada este año cuando el diario de circulación nacional, La Jornada, publicó uno de los exámenes que se iban a aplicar, lo que motivó que se suspendiera y pospusiera la aplicación del examen ya programado.

$12 \mathrm{Al}$ arribo al poder en el país de un partido diferente al PRI se llegó a hipotetizar la posibilidad de que el nuevo gobierno asumiera una relación diferente con los sindicatos oficialistas, cosa que no sucedió (DE LA GARZA, 2000).

13 Sin embargo, la CNTE siguió creciendo hasta lograr ganar estatutariamente la representación de secciones enteras como la XXII de Oaxaca, que se convirtió en el contingente más consolidado de la CNTE, la sección VII de Chiapas, la cual posteriormente fue recuperada por los institucionales y para 1998 volvió a ser dirigida por maestros afines a la CNTE. Otros contingentes en Valle de México, Guerrero, Morelos, Hidalgo y otros llegaron a tener agrupados a sectores muy representativos de maestros aunque no lograron consolidarse como representaciones oficiales.

14 Este tipo de liderazgo es más afín al grupo de 'los modernizadores en el poder, en cambio el grupo VRM sostenía una visión de la política menos moderna, más patrimonialista.

15 En las nuevas condiciones de reestructuración económica y social instauradas en México desde 1982, Jonguitud, al igual que el 'líder histórico de los petroleros', Joaquín Hernández Galicia representaban liderazgos onerosos en términos de las nuevas realidades políticas, razón por la cual fueron desplazados del poder.

16 Vale la pena recalcar que ese tipo de funcionamiento es un ideal y que no necesariamente tal forma de democracia se practica en todo momento.

\section{Referências}

ALARID, D. Reestructuración de la educación básica y proceso democrático en la sección del SNTE. In: De la Garza, E. (Coord.). 
Democracia y cambio sindical en México. México: UAM- Y - Plaza y Valdez, 2001.

APPLE, M. Maestros y textos. Barcelona: Paidós, 1989.

Educación y poder. Barcelona: Paidós, 1995.

ARONOWITZ, S. The knowledge Factory. Boston: Beacon Press, 2000.

ARNAUT, A. La evolución de los grupos hegemónicos en el SNTE. México: CIDE, 1992.

ARNAUT, A. Historia de una profesión. Los maestros de educación primaria en México, 1887-1994. México: CIDE, 1996.

ARNAUT, A. La federalización educativa en México: 1889- 1994. México: SEP, 1998.

BALL, S. La micropolitica de la escuela. Barcelona: Paidós, 1989.

Foucault y la educación: disciplinas y poder. Madrid: Morata, 1997.

BANCO MUNDIAL. El financiamiento de la educación en los paises en desarrollo. Washington: Banco Mundial, 1995.

BONAL, X. Globalización y política educativa: un análisis crítico de la agenda del Banco Mundial para América Latina. Revista Mexicana de Sociologia, México (D.F.), v. 64, n. 3, jul./ sept. 2002.

BOURDIEU, P. Sociología y cultura. México: CNCA- Grijalbo, 1990.

COLL, C. et al. Desarrollo psicológico y educación II: psicología de la educación. Madrid: Alianza, 1990.

CONTRERAS, J. La autonomía del profesorado. Madrid: Morata, 1997.

COOK, L. Organizing dissent: unions, the state, and the democratic teachers' movement in Mexico. Pennsylvannia: PSU Press, 1996.

DÍAZ BARRIGA, A. Universitarios: institucionalización académica y evaluación. México: CESU- UNAM, 1997.

Carrera magisterial y evaluación de profesores: entre lo administrativo

y lo pedagógico en M. Rueda y M. Landesmann, ¿Hacia una nueva cultura de la evaluación de los académicos? México: CESU/UNAM, 1999. 
GALINDO C., J Técnicas de investigación en sociedad, cultura y comunicación. México: Addison Weslley Logran, 1998.

DE LA GARZA, E. Ascenso y crisis del estado social autoritario. México: COLMEX, 1988.

- (Coord.). Crisis y sujetos sociales en México. México: Porrua, 1992.

- (Coord.). Políticas públicas alternativas en México. México: La

Jornada- CIICH-UNAM, 1996. Valdés, 2000a.

(Coord.). La formación socioeconómica neoliberal. México: Plaza y

GIDDENS, A. Las nuevas reglas del método sociológico. Buenos Aires: Amorrortu, 1990.

. La constitución de la sociedad. Buenos Aires: Amorrortu, 1995.

GIMÉNEZ, G. La teoría y el análisis de la cultura. Problemas teóricos y metodológicos. In: GONZÁLEZ; GALINDO, J. (Coord.). Metodología y cultura. México: CNCA, 1994.

GIROUX, H. Educación y poder. Barcelona: Paidós, 1990.

GRAMSCI, A. Cuadernos de la cárcel. México: Juán Pablos, 1975. Tomo 1.

HARGREAVES, A .Profesorado, cultura y postmodernidad. Cambian los tiempos cambia el profesorado. Madrid: Morata, 1996.

HARVEY, D. The condition of posmodernity. Balden, USA: Blackwell, 1990.

IBARROLA, M. et al. ¿Quiénes son nuestros maestros? México: Fundación SNTE, 1997.

JACKSON, P. La vida en las aulas. Madrid: Morata, 1996.

JIMÉNEZ, L. La reestructuración de la escuela y las nuevas pautas de regulación del trabajo docente. Revista mexicana de investigación educativa, v. 8, n. 19, sept./dic. 2003.

MÉXICO SECRETARÍA DE EDUCACIÓN PÚBLICA (SEP).

Lineamientos Generales de Carrera Magisterial. México: SEP, 1998.

MÉXICO. PODER EJECUTIVO FEDERAL. Acuerdo Nacional para la

Modernización de la Educación Básica y Normal (ANMEB). México: PEF, 1992.

PERSPECTIVA, Florianópolis, v. 26, n. 2, 433-456, jul./dez. 2008

http://www.perspectiva.ufsc.br 
MÉXICO. PODER EJECUTIVO FEDERAL. Plan nacional de desarrollo: 1977- 1982. México: PEF, 1977.

MÉXICO. PODER EJECUTIVO FEDERAL. Plan para la modernización educativa: 1989- 1994. México: PEF, 1989.

POPKEWITZ, T. S. Sociología política de las reformas educativas. Madrid: Morata, 1998.

SANDOVAL, E. Los maestros y su sindicato: relaciones y procesos cotidianos. México: DIE-CINVESTAV, 1997.

SCHMELKES, S. La calidad en la educación primaria. México: SEP/FCE, 1997.

SMYTH, J. Teachers Work in a Globalizing Economy. Londres: Falmer Press, 2000.

STREET, S. Maestros en movimiento. México: CIESAS, 1992.

STREET, S. "El movimiento magisterial como sujeto democrático ¿autonomización de los educadores o ciudadanización de la educación?". El cotidiano 87, México, v. 14, n. 8, 1998.

STREET, S. Trabajo docente y poder de base en el sindicalismo magisterial en México. en: La Ciudadanía Negada. Buenos Aires: CLACSO, 2000.

STREET, S. Trabajo docente y reforma escolar en América Latina. Washington, USA: [s.n.], 2001. Documento presentado en encuentro de la Latin American Studies Asociation.

TEDESCO, J. C. Nuevas estrategias de cambio educativo en América Latina. In: TEDESCO, J. C. et al. Necesidades básicas de aprendizaje: Estrategias de acción. Santiago de Chile, UNESCO/IDRC, 1993.

THOMPSON, J. Ideología y cultura moderna. México: UAM- Xochimilco, 1993.

WILLIS, P. Aprendiendo a trabajar. Madrid: Akal, 1988.

WOODS, P. La escuela por dentro. Barcelona: Paidós, 1987. 


\section{Elementary School Teachers in Mexico (DF) and the res- tructuring of the educational sector}

\section{Abstract:}

This study analyzes how the "modernizing" educational policies restructured education in Mexico, reconfiguring the working conditions of elementary school teachers and how they conduct their jobs. The restructuring underway has continued in agendas set by international agencies such as the World Bank, the International Monetary Fund, and others, under new policies of differential pay for the work of teaching. The paper analyzes the strategies elementary school teachers in Mexico City have used to confront what they experience as negative consequences of education reform for their work and public education.

Key words: Education-Mexico. Educational reform-Mexico.

\section{Os professores da Educação Básica em México (DF) fren- te a reestruturação do Setor educacional}

\section{Resumo:}

No presente texto se aborda a reestruturação no setor educacional no México e as formas em que se manifestaram, traduzidas, estas, na implementação das políticas educacionais "modernizadoras", que incidiram na reconfiguração das condições de trabalho dos professores da Educação Básica em México e como esta afetou as tarefas dos mesmos. A reestruturação em andamento seguiu as pautas marcadas pelos organismos internacionais como o Banco Mundial, (BM) o Fundo Monetário Internacional (FMI), e, entre outras questões, as novas políticas de redistribuição do trabalho docente. Também, se analisam as estratégias com que os professores da educação básica da cidade de México enfrentaram as conseqüências negativas que vivenciaram da reforma educativa para seu trabalho e para a educação pública.

Palavras-chave: Educação-México. Reforma Educacional-México.

José David Alarid Dieguez

Dirección: Carretera al Ajusco \# 24, Col. Héroes de Padierna, Tlalpán, México, D. F.

Teléfono y fax UPN: 5630-9747, extensiones. 1419 y 1517

E-mail:montgal3@yahoo.com.mx

Recebido em: 10/11/2008

Aprovado em: 15/11/2008 